\title{
Cell Microenvironment
}

National Cancer Institute

\section{Source}

National Cancer Institute. Cell Microenvironment. NCI Thesaurus. Code C48199.

The complex relationships between tumor cells and the neighboring cells in the host

environment. Cellular signaling within the microenvironment can promote the continuing survival and growth of tumor cells, or apoptosis (cell death). Research is currently underway to manipulate this relationship by altering the host environment in ways that silence or inhibit pro-survival signals concurrent with standard therapies. 